UDC 811.135.1'366.52

DOI 10.18485/primling.2015.16.3

\title{
Constantin-Ioan Mladin
}

\author{
Université « 1 Decembrie $1918 »-$ Alba-Iulia (Roumanie) \\ Université « Sts. Cyril et Méthode »-Skopje \\ (République de Macédoine)
}

\section{LE GENRE NEUTRE DES NOMS ROUMAINS EN CONTEXTE ROMAN ET BALKANIQUE}

Résumé: Cette communication se donne pour but de réfléchir sur un aspect insolite de la morphologie roumaine - le genre neutre des noms.

De façon générale, on accepte par consensus que de toute les langues néolatines c'est seulement le romain qui a conservé le neutre en tant que catégorie grammaticale consistante et productive, le neutre s'assumant, à côté du sous-genre personnel, le rôle d'agent de ré-motivation du genre naturel. Par surcroît et malgré les prédictions pessimistes de quelques linguistes du siècle dernier qui anticipaient la désintégration du neutre, celui-ci s'avère un genre très dynamique et moderne dans le roumain actuel.

Pour mieux comprendre le statut et la provenance du neutre roumain, cette présentation : 1) va retracer d'une manière succincte et critique les principales théories élaborées sur ses origines possibles (filiation avec le latin, parenté avec l'albanais, influence du vieux slave) et 2) va mettre en rapport le neutre de la langue roumaine, langue simultanément romane et balkanique, avec des situations identiques ou similaires qui existent dans d'autres langues contemporaines dont le roumain est historiquement apparenté (l'italien et le romanche, le français, l'espagnol).

Cette recherche se propose aussi de diagnostiquer l'efficacité (réelle ou apparente) des différentes méthodes utilisées pour la juste reconnaissance du neutre roumain, aspect assez problématique et difficile à surmonter lors de l'apprentissage du roumain en tant que langue étrangère (la perspective des apprenants francophones a été particulièrement privilégiée).

Mots-clés: genre grammatical (masculin, féminin, neutre), genre naturel, langue albanaise, langue espagnole, langue française, langue italienne, langue latine, langue roumaine, mots androgynes, romanche, vieux slave (vieux-bulgare).

1. Introduction à la problématique du genre grammatical. Le genre, l'une des catégories grammaticales les moins logiques, a attiré la curiosité de tous : philosophes et linguistes, psychanalystes ou simples observateurs du langage et des langues (Arrivé 1997 : 81). Cet intérêt particulier qu'on lui a accordé est motivé par la relation directe 
que le genre contracte avec la sexualité aussi bien que par son aspect peu fonctionnel ${ }^{1}$ (ibidem, 93).

Les difficultés que doivent surmonter les locuteurs, natifs ou non, lors du processus de l'acquisition du genre sont directement liées aux aspects suivants :

1) Sémantiquement, le poids du genre reste assez insignifiant, parce que, le plus souvent, le genre reste immotivé, il n'est donc qu'une catégorie vide et purement mécanique dont l'enjeu serait constitué par de simples faits d'accord (Violi 1987 : 18)². Aussi paradoxal que cela puisse paraître, cette réalité se vérifie même dans la correspondance défaillante qui existe entre le genre grammatical et genre naturel (le sexe) dans la classe des animés (Arrivé 1997 : 90). Il suffit de rappeler dans ce sens que sur 7800 noms d'animaux qui existent en français 6100 sont seulement masculins et 1700 seulement féminins. Alors qu'il n'y a que 23 espèces d'animaux qui disposent de formes désignant l'opposition de sexe (Dubois 1989 : 88). Pas toujours facile à repérer et à mémoriser celles-ci par-dessus, même par un locuteur natif ${ }^{3}$.

2) Par contre, le genre se relevé d'une importance décisive au niveau syntaxique puisqu'il est tenu directement responsable d'assurer la cohérence de l'énoncé ${ }^{\text {(Arrivé }}$ 1997 : 86).

3) En général, pour déterminer le genre auquel un nom appartient (surtout dans le cas des noms inanimés), il faut corroborer les indices fournis au moins par les deux sinon par les trois critères suivants (Sterpu 2012 : 95) :

a) le critère sémantique, qui suppose l'encadrement sémantique du nom dans une classe quelconque ;

b) le critère formel, qui consiste dans l'assignation des noms (agissant comme « donneurs » $\mathrm{d}^{\prime}$ accord $^{5}$ ) à tel ou tel genre à l'aide de leur terminaisons de singulier et de pluriel ;

c) le critère distributionnel, qui se concrétise par la prise en compte des formes que revêtent les déterminants des noms (choix des articles et des pronoms-substituts, accord des adjectifs-attributs - mots satellites (devenus « receveurs » d'accord ${ }^{6}$ ).

Il est vrai qu'on peut toujours indiquer quelques marques spécialisées pour les reconnaître les genres (critère formel), sauf que leur grand nombre et leur occurrence à plus d'un genre rendent ces distinctions peu utilisables :

1 Voir les variations considérables qui affectent cette catégorie selon les langues.

2 Cela n'empêche pas qu'il soit investi dans certains cas d'une valeur sémantique.

3 Voir à ce sujet un extrait du récit de Jean-Luc Lécaillé $(2010: 30)$ ou l'auteur remémore d'un humour succulent une leçon de grammaire pendant sa scolarité : «Il fallait aussi affronter certains mots au genre douteux : 'ivoire' et 'enclume' par exemple. Je pense aussi à 'argile, épitre et orge' qui sont tous du sexe... féminin. Pas toujours facile de s'y retrouver. D'ailleurs, pourriez-vous me préciser le genre de ces cinq mots relatifs à la maladie ? : 'urticaire, aphte, escarre, dartre, acné'. Et le genre de ces cinq fleurs ? : 'colchique, iris, narcisse, azalée, ellébore' (alors là, on fait moins malins). / Pour embrouiller le tout, il y avait les mots androgynes : ceux qui changent de genre selon qu'ils sont au singulier ou au pluriel. Comme l'amour. L'amour est masculin au singulier et féminin au pluriel. Moi je dis que l'amour n'est ni masculin ni féminin. Il est. Il est ou il n'est pas. Il est particulier, unique et rebelle. Il est le plus beau des plus... belles !!! (Ah non, dans ce cas-là on dit : le plus beau des plus beaux, c'est un des délices de la langue française. Attention « délice » est un mot androgyne également tout comme : orgue !). ».

4 Par le biais de l'accord et de la correspondance avec les pronoms correspondants.

5 Selon la terminologie de D. Elmiger (Elmiger 2015).

6 Idem. 
a) Principales terminaisons des mots masculins français : -age (chômage), -as (bras), -d (poignard), -eau (moineau), -et (coffret), -is (cliquetis), -isme (socialisme), -l (vitrail), -ment (sentiment), -oir (miroir), -ot (angelot), -r (pommier), -ron (chaperon), - $t$ $($ mot $) \ldots$

b) Principales terminaisons des mots féminins français : -ade (pommade), -aille (bataille), -ance (balance), -asse (paillasse), -ée (cheminée), -elle (poubelle), -ence (essence), -ère (cuillère), -esse (finesse), -ette (starlette), -eur (douleur), -euse (coiffeuse), -ie (maladie), -ine (tartine), -ise (maîtrise), -ison (prison), -itude (exactitude), -ose (névrose), -otte (menotte), -sion (pension), -té (qualité), -tion (révolution), -ure (brûlure)...

c) Principales terminaisons des mots masculins roumains : $c_{1}$ ) consonne non-palatalisé (băiat « garçon »), $\mathrm{c}_{2}$ ) consonne palatalisé (ochi « œil »), $\mathrm{c}_{3}$ ) voyelles et semi-voyelles: - $\breve{a}$ (popă « prêtre, curé »), -e (câine « chien »), - ěa (Oprea - nom propre), $-a$ (Toma - nom propre), $-\breve{l}$ (pui « petit - jeune animal »), -u (membru « membre »), $-\breve{u}$ (erou « héro »)...

d) Principales terminaisons des mots féminins roumains : $d_{1}$ ) consonne non-palatalisé (luni « lundi »), $\mathrm{d}_{2}$ ) voyelles et semi-voyelles : -á accentué (cazma « bêche »), - $\breve{a}$ (cămașă « chemise »), -e (floare « fleur»), - $\breve{a}($ cafea « café »)...

e) Principales terminaisons des mots neutres roumains : $e_{1}$ ) consonne non-palatalisé (creion « crayon »), $\mathrm{e}_{2}$ ) consonne palatalisé (unchi « oncle »), $\mathrm{e}_{3}$ ) voyelles et semi-voyelles : -e (spate « dos, région dorsale »), $-i$ (zi « jour »), $-\breve{l}$ (joi « jeudi »), -o (radio « radio »), $-u$ (lucru « objet, chose »), $-\breve{u}($ tablou « tableau »), -ú accentué (tabu « tabou »)...

D'autre part, il est vrai aussi que, en dépit d'un nombre accablant d'exceptions, il y a quelques indices sémantiques qui permettraient un certain regroupement des noms par classe de genre (critère sémantique). Par exemple :

a) Sont plutôt masculins : les noms français de couleurs, de langues, d'arbres, de métaux, de jours, de mois, de saisons, des points cardinaux, de figures géométriques, du système métrique, des lettres de l'alphabet, des termes grammaticaux et de linguistique, les noms d'êtres imaginaires ou mythiques... et les noms roumains d'arbres, de légumes, de plantes et de fleurs, de mois, de lettres de l'alphabet...

b) Sont plutôt féminins : les noms français de sciences, de disciplines ou d'arts... et les noms roumains de saisons, de monnaies, de notes de musique, de langues, les noms abstraits désignant des caractéristiques physiques, morales ou bien des états d'âme et des sentiments...

c) Sont plutôt neutres : les noms roumains désignant des inanimés et des abstractions, les noms de jeux et de sports, les noms de vents...

Le critère distributionnel, quant à lui, n'est qu'un moyen de vérification du genre, parce que le choix des « receveurs » d'accord suppose la connaissance préalable du genre des « donneurs » d'accord.

Pour conclure, il faut admettre donc que, malgré un bon nombre d'indices d'ordre formel et sémantique, le genre des noms reste rarement prédictible. Et cela parce qu'il varie en fonction de son étymologie et d'une suite très peu contrôlable de conventions d'usage (variables d'une époque à une autre, d'un milieu social à un autre, d'un espace géographique à un autre). Néanmoins, les grammaires ont tenté de décrire et de regrouper les noms dans des classes de genre selon le critère formel (à l'aide de désinences) en postulant que, normalement, le même suffixe est toujours associé à un même genre. 
Cette classification s'avère de loin plus efficace en français qu'en roumain où pas mal de suffixes sont communs au moins à deux genres sinon à tous les trois - surtout au masculin et au neutre (Avram $1997^{2}$ : 48). Et cet aspect devient encore plus embarrassant pour les locuteurs ayant une autre langue maternelle (Papp 1978 : 473). Pour le roumain, par exemple, la seule règle qui pourrait avoir une valeur générale dit que tout nom qui finit par une consonne ne peut être que masculin ou neutre et jamais féminin. Mais la réci-

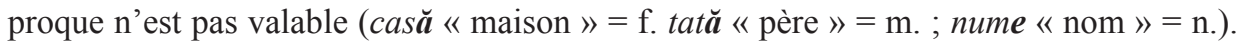
De telles inconséquences existent également en français : le suffixe -eur, spécifique aux noms féminins qui dénomment une sensation ou une chose (peur, fleur) peut être assumé aussi par des noms masculins (cœur, honneur).

4) À la différence des autres catégories grammaticales, le genre se présente comme un trait inhérent et fixe du nom, solidaire, en roumain et en français, des morphèmes de nombre, de détermination et de cas (ce dernier, en roumain). Grâce à cette spécificité du genre, dans le cadre de l'apprentissage d'une langue, ladite catégorie grammaticale revêt une importance particulière, dont dépendent toutes les autres catégories grammaticales déjà mentionnées (Sterpu 2012 : 96).

2. Le neutre dans les langues romanes. Connu par la majorité des langues descendant de l'indo-européen (le grec, le latin, les langues slaves, les langues germaniques), le neutre s'est volatilisé dans les langues romanes occidentales. Tandis que la distinction masculin vs. féminin s'est généralisée et s'est imposée presque partout dans les langues issues de l'indo-européen, la distinction animé vs. inanimé, sauf quelques exception (les langues slaves, le roumain...), a quasiment disparu (Violi 1987 : 16).

D'une façon habituelle, on estime que de toutes les langues romanes c'est seulement le roumain qui a réussi à sauvegarder le neutre latin en tant que catégorie grammaticale solide et productive, ce genre illustrant, à côté du sous-genre personnel, une certaine tendance de ré-motivation du genre grammatical, à savoir une meilleure correspondance avec le genre naturel (DGȘ $1997^{1}:$ 217-218, 324).

Malgré ce qui vient d'être dit, le neutre ne s'est pas conservé seulement en roumain, des traces de ce genre pouvant être détectées aussi dans d'autres langues romanes (Bujor 1955; 61-62, 647\% Pătruț 1956 : 38 ; Livescu 2009 : 2647).

2. 1. Les traces du genre neutre en italien. En italien et en romanche, langues moins novatrices que les autres idiomes occidentaux (Bujor 1955; 62 ; Bonfante 2001 ; 124 ; Găitănaru 2007 ; 145, 147) :

7 « Il n’y a pas de genre neutre ou hétérogène en roumain. Des noms masculins au singulier et féminins au pluriel existent aussi en italien. Cette particularité des deux langues émane des lois phonétiques qui ont présidés le passage du latin vulgaire aux langues romanes respectives, et tout rapprochement des langues des peuples voisins, qui ne connaissent pas cette situation, est exclu. » (« În limba română nu există genul neutru, ori eterogen. Particularitatea de a avea substantive cu genul masculin la singular și genul feminin la plural o are și limba italiană, ambele limbi posedând-o pe baza legilor fonetice care au prezidat trecerea limbii latine vulgare în limbile romanice respective, cu aceasta neavând nicio legătură limbile popoarelor învecinate, care nu cunosc această situație. »). 
1) quelques traces du paradigme du genre neutre latin ont survécu, toujours dans une perspective régressive. Il s'agit de quelques noms collectifs et quelques pronoms personnels ${ }^{8}$ : lat. n. cornu $«$ corne »> it. m. sg. corno - f. pl. corna; lat. n. $\breve{s} «$ os »> it. m. sg. os - féminin pl. ossa ; lat. n. ovvum > it. m. uovo « œuf»-f. pl. uova $)^{9}$;

2) il y a un suffixe (-ora / -ura) qui correspond au suffixe roumain -uri pour les noms féminins, surtout dans le registre archaïque et dialectal : corpo « corps » / corpura, foco / fuoco « feux » / focura / fuocora.

2. 2. Le « neutre » en espagnol. En espagnol aussi il y a une sorte de neutre exprimé seulement à l'aide de l'article défini $l o$, auprès des noms postadjectivaux dénotant des concepts abstraits : lo bueno « ce qui est bon », lo contrario « le contraire de qqch », lo externo « ce qui est extérieur à qqch», lo importante « ce qui est important», lo malo «le mal», lo profundo « ce qui est profond».

2. 3. Le « neutre » en français. Un tas d'oscillations d'interprétation et d'incertitudes peuvent être constamment constatées à l'égard de la catégorie du neutre français (Marchello-Nizia 1989: 173).

Le genre des noms en français est un héritage latin et si le neutre proprement-dit fait défaut dans le français moderne c'est qu'il s'est affaibli dès le bas latin et a disparu dans le latin populaire / parlé. Au moins deux facteurs pourraient se trouver à l'origine de ce processus :

1) Si le masculin et le féminin peuvent être mis en rapport direct avec le genre naturel, le neutre est dépourvu d'un tel correspondant (templum « temple » est neutre mais dŏmŭs « maison » est féminin). Du coup, le neutre du latin n'avait pas de fonction sémantique assez cohérente et homogène pour justifier sa présence dans le français.

2) La déclinaison des noms neutres latins au pluriel est très proche de celle du masculin (à part pour la classe des noms de la 3e déclinaison en -en : nōměn, flūměn dont l'accusatif reste nōměn, flüměn, alors que les masculins de la $3 \mathrm{e}$ déclinaison ont un accusatif en $-e(m)$, avec un $-m$ labile). Au singulier, pour les noms de la 2e déclinaison, le neutre se distingue du masculin seulement au nominatif-vocatif (templum; dŏmĭnus), pour tous les autres cas la forme restant identique (templum, templi, templo ; dömı̆num, dŏmı̆ni, dömı̆no).

Dans l'ancien français (tout comme dans le provençal ancien, l'italien de l'est et l'espagnol) il y avait un certain nombre de noms neutres en -e (brasse, charre, corne, cote, leigne, membre, ossemente... ; cf. ambes les brace « les deux bras ») (Marchello-Nizia 1989 ; 174 ; Bonfante 2001 : 126, apud Găitănaru 2007 : 146), un nombre insuffisant quand même pour en constituer une classe à part entière. Faute de neutre, le français a redistribué les neuf dixièmes des noms hérités du latin dans la catégorie du masculin, plus proches morphologiquement du neutre que du féminin latin.

8 Dans les dialectes italiens septentrionaux, ces noms reçoivent au pl. la terminaison - $e$, tous comme en roumain.

9 À ceux-ci s'ajoutent quelques noms masculins latins qui ont subi le même traitement en italien : lat.

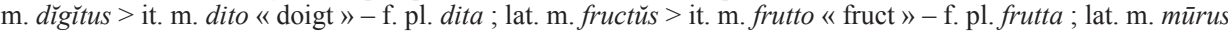
$>$ it. m. muro « mur, paroi »- f. pl. mura. 
Il n'est pas exclu cependant qu'un nom neutre latin passe au féminin, vu la confusion entre la désinence neutre du pluriel en $-a$ ou $-i a$ (sg. n. de templum = pl. templa) avec la flexion des noms féminins en - $a$ de la 1re déclinaison (rosa,-ae). C'est ainsi que des noms neutres latins sont devenus féminins en français (Picoche - Marcello-Nizia 1993 : 16 ; Guillard-Chamart 2009 : 16 ; Michel 2015) : lat. fŏlŭum - fŏlı̆a > sg. fr. feuille.

À côté des noms clairement repartis entre le masculin et le féminin, le français actuel enregistre un certain nombre de noms à usage soit hésitant, soit à genre douteux ${ }^{10}$ :

1) Certains noms changent de genre selon l'emploi : automne (masculin quand l'adjectif le précède : un bel automne, féminin quand l'adjectif le suit immédiatement : cette automne délicieuse ${ }^{11}$ ) ; chose (cette grande chose vs. quelque chose de grand); espace (masculin : l'espace infini, un espace exigu, sauf en typographie « lamelle qu'on intercalait entre les caractères de plomb » : espace fine, espace forte) ; hymne (féminin en parlant des hymnes qu'on chante dans l'église - poème qui célèbre la gloire de Dieu... : l'hymne pascale et au masculin dans les autres sens : " chant ou poème composé en l'honneur d'un dieu ou d'un héros, chez les Anciens ; chant ou poème lyrique célébrant une personne, un sentiment, un événement » : hymne grec à Apollon; hymne national ; réglisse (féminin quand on désigne la plante, masculin quand on désigne la racine, le jus ou la pâte qu'on en tire) < relâche (masculin, sauf en termes de marine).

2) D'autres noms apparaissent en variation libre soit au masculin, soit au féminin, sans aucune implication sémantique : agave (agave américain / américaine); aigle (aigle altièr / altière).

3) Trois noms (amour, délice et orgue) disposent d'un double genre en fonction du nombre. Ces noms sont appelés parfois mots androgynes (TLFi ; LAROUSSE, Grevisse \& Goosse : § 482) : sg. m. un amour fervent ${ }^{12}$, un amour heureux, un premier amour - $\mathrm{pl}$. f. Mais le vert paradis des amours enfantines ${ }^{13}$, des amours heureuses, de belles amours ; sg. m. un délice - pl. féminin des délices infinies ${ }^{14} ; \mathrm{sg}$. m. un orgue du XVIIe siècle - pl. f. de belles orgues, les grandes orgues de Notre-Dame de Paris - lorsqu'il s'agit d'un orgue d'une valeur unique.

C'est particulièrement cette dernière et minuscule sous-classe qui est très proche de la classe des noms neutres roumains. Toute proportion gardée, cette situation pourrait être mise en relation avec le genre neutre roumain surtout si on évoque les deux autres appellations dont on désigne parfois celui-ci : ambigenre / roum. ambigen et genre hétérogène / roum. eterogen (voir supra).

2. 4. Le neutre roumain. Des trois genres qui existent en roumain c'est le neutre qui est le plus homogène (Avram 1997² : 47 ; Irimia 1997 : 47 ; DGȘ 1997² : 324). Celui-ci réunit des noms qui désignent : 1) des inanimés - c'est la sous-classe la plus consistante (creion « crayon », palton « paletot»);2) l'espèce - des noms placés en de-

10 Souvent c'est la finale en -e qui influence l'encadrement de ces noms dans la classe du féminin. Certaines formes de ces couples sont considérées vieilles ou vieillies. Cependant l'usage reste très flottant (Grevisse \& Goosse : § 482).

$11 \mathrm{Si}$ un adverbe ou un verbe se trouve entre ce nom et l'adjectif, automne est du genre masculin (l'automne est beau).

12 Le féminin singulier (une belle amour) en cache des intentions stylistiques.

13 Charles Baudelaire, Moesta et errabunda.

14 Précédé par de, le mot garde sa forme masculine (un des, un de, le plus grand des : un des plus grands délices). 
hors de l'identité de genre (animal « animal », mamifer « mammifère »); 3) des entités collectives (batalion « bataillon », popor « peuple »).

À côté des désinences communes aux deux autres genres, le neutre se sert d'une désinence pour le pluriel qui semble lui être spécifique (-uri : cornuri « croissants», lucruri « choses »), très répandue aussi parmi les néologismes (pixuri « stylos-billes », stilouri «stylos »).

Cette désinence, propagée d'ailleurs aux noms féminins (sg. blană « fourure » - pl. blănuri, sg. mătase « soie » - pl. mătăsuri « différentes sortes de soie ») a acquis le statut de marque emblématique du neutre étant souvent invoquée comme argument suprême et incontestable de l'existence de ce genre en tant que catégorie à part entière et opposable tant au masculin qu'au féminin.

Ce n'est pas autant l'existence elle-même du neutre dans le roumain qui intrigue, mais son curieux comportement morphologique, qui le distingue aussi bien du neutre latin que du neutre des langues slaves : «Bizarre catégorie, du point de vue logique, car c'est tout à fait inconcevable que lorsque l'objet est un [s. a. ], il soit masculin, mais que dès que les objets sont deux, trois [s. a. ], etc. , ils deviennent féminins. » (Tănase 1974 : 331). Cette bizarrerie consiste donc dans le fait qu'au singulier les noms neutres se comportent comme des masculins et qu'au pluriel ils se convertissent au féminin. Les autres appellations sous lesquelles ce genre est connu d'ailleurs semblent plus transparentes, plus motivées à cet égard : ambigenre (roum. ambigen - Pătruț, 1974) et genre hétérogène (roum. eterogen - Goga 1966 : 310).

Un phénomène semblable mais à une échelle beaucoup plus réduite a été signalé aussi en tchèque. Il s'agit de trois noms qui changent de genre lors du passage du singulier, où ils sont neutres, au pluriel, quand ils deviennent féminins (Hořejši $1957: 415$ ) : ditko « gamin, bébé » (< ditě « enfant »), oko « œil », ucho « oreille».

2. 4. 1. Histoire du neutre roumain. Pour ce qui est des origines du neutre roumain, elles sont encore loin d'être complètement et définitivement élucidées. Plusieurs théories ont été élaborées à ce sujet :

2. 4. 1. 1. Le neutre roumain vs. le neutre albanais. Le neutre roumain doit être mis en relation avec le substrat thraco-dace, suite d'un examen en parallèle avec la langue albanaise (Sandfeld 1930 ; Tøgeby 1952 ; Nandris 1961 ; Brâncuş 1963 ; Iordan - Robu $1978: 370$; Bonfante $2001: 136$ ).

À côté du masculin et du féminin, l'albanais connaît aussi une classe hétérogène sémantiquement et en régression (alb. gjinia asnjanëse) qui rassemble environs 15 noms neutres dont seulement 5 sont acceptées par la langue littéraire (Feuillet $2001: 1516^{15}$; Veselaj 2012), marqués au niveau de l'expression - noms terminés en -e ou en - $a$ en fonction de la variation dialectale : bukëra «bouchées de pain sec»-pluralia tantum, dhe « terre », krahtë « epaule », leshtë « laine », mjaltë « miel », rrobëra « costumes, ensembles de différents pièces d'un même habillement », sytë « œil », ujë « eau », veshë « oreille »...

Il s'agit de noms majoritairement concrets et rarement abstraits (GGSh 2002 : $92)$ - alb. personazhe kryesore « personnages principaux, protagonistes $»^{16}$ qui peuvent

15 En revanche, environs 70 noms neutres proprement-dits (non-dérivés) ont pu être recueillis des textes anciens (Veselaj 2012).

16 Sauf deux noms désignant des organismes minuscules : alb. baktere të dëmshme « bactéries nuisantes », alb. helmuese insekte « insectes venimeux ». 
recevoir au pluriel des déterminants féminins (Sandfeld 1930 : 139 ; Габинский 1956 : 86-87 ; Kokona 2002 ; DAR 2003 ; Găitănaru 2007 : 146-147) : alb. pallat « chateau »pallatë, alb. vënd «lieu »-vënde.

Ayant en vue les similarités frappantes saisies entre les neutres roumains et albanais et compte tenu de la productivité nettement supérieure de la formation du pluriel roumain à l'aide des désinences - $e$ et -uri / -(ë)ra, par rapport à l'albanais, on a soutenu même que c'est le thraco-albanais qui a empruntée ce genre du roumain (Bonfante 2001 :136).

Enfin, d'autres linguistes ont minimisé ou ont nié toute influence possible du substrat dans la sauvegarde du neutre roumain. Ou bien, on a considéré que c'est plutôt l'influence exercée par le latin oriental sur le roumain et sur l'albanais qui pourrait expliquer la liaison entre les neutres des deux langues (Pătruț $1956: 38$ ).

2. 4. 1. 2. Le neutre roumain vs. le neutre vieux slave. En outre, on a avancé l'idée que c'est sous l'influence du vieux slave que le roumain a pu conserver le neutre latin qui était en train de se désagréger dès le bas latin (Iordan 1956² :239).

Par ailleurs, une possible influence du vieux slave sur le neutre roumain a été rejetée en vertu du traitement subi par les emprunts du vieux slave en roumain, ceux-ci étant absorbés par les classes des noms de provenance latine qui s'étaient déjà constituées en roumain (ibidem : 31-33; Pătruț $1974: 133-153)$ :

1) les neutres slaves avec le thème en -o sont entrés dans la classe des féminins, le roumain ne disposant pas d'une catégorie de noms en -o, comme le vieux slave : sl. greblo « rateau » > roum. greblă, ar. griblă, megl. greblă, sl. kopyto « sabot» > roum. copită, ar., megl. cupită. Tous ces emprunts ont été traités comme des noms slaves avec le thème en $-a-:$ sl. kosa « faux » > roum., ar. , megl. coasă, sl. lopata « pelle » > roum. lopată, ar., megl. lupată ;

2) les neutres slaves avec le thème en -e sont entrés dans la classe des féminins en -e : sl. podŭgorije « vignoble » > roum. podgorie, sl. prěmeždije « peril, danger » > roum. primejdie ;

3) les neutres roumains de provenance slave sont masculins dans cette langue : sl. biči « fouet » > roum. bici, sl. briči « rasoir » > roum. , megl. brici;

4) quatre noms slaves seulement ont conservé leur genre en roumain : v. sl. bliudŭ « terrine » > roum. blid, v. sl. klopotü « cloche » > roum. clopot, v. sl. jezerŭ « lac alpin » $>$ roum. iezer, v. sl. maslo « huile» > roum. maslu « messe pour une personne gravement malade ou pour la consécration de l'huile sacrée $»$.

2. 4. 1. 3. Le neutre roumain vs. le neutre latin. Le genre neutre est considéré un héritage direct du latin vulgaire. Le processus de formation de cette catégorie de noms s'est prolongé dans la romanité danubienne et balkanique et ensuite en roumain. Il est évident donc que le slave n'a pas pu contribuer au maintien et au soutien du neutre latin en roumain, parce que (Pătruț $1956: 38): 1$ ) le neutre roumain est complètement différent du neutre du vieux slave ; 2 ) les noms neutres slaves sont entrés en roumain dans la classe des féminins et non pas dans celle des neutres, les neutres roumains de provenance slave étant des masculins dans la langue d'origine.

Le sens du neutre a commencé à s'assombrir dans le latin vulgaire, suite à la confusion avec le masculin et parfois même avec le féminin, comme résultat de la disparition des consonnes finales $-s$ et $-m$ qui a effacé la distinction entre le masculin et le féminin (Ivănescu $1980: 301$ ). Néanmoins, comme il est difficile à accepter que tous ces noms ont cessée d'appartenir pour un certain temps à un genre quelconque, même si ce 
n'était que du point de vue formel, le neutre n'a pas pu totalement s'évanouir dans le latin de la Romania orientale. Parce que, une fois perdu le modèle de ses oppositions caractéristiques, il n'aurait pas pu se recréer après (Coteanu 1969 : 36 ; Găitănaru 2005 : 605).

Mise à part cela, la moitié des neutres issus du latin étaient eux aussi des neutres dans le latin classique (Doca - Rochetti 1992 : 56-57 ; Găitănaru 2005 : 605) : roum. cap - capete < lat. căput « tête », roum. scaun - scaune < lat. scamnum « chaise ».

Dans le bas latin, le neutre avait tendance à incorporer les inanimés et a acquis la désinence -ora, l'accord avec l'adjectif se faisant au féminin. La catégorie sémantique s'est conservée dans la conscience des locuteurs de la basse latinité et de langues romanes où c'est seulement la catégorie formelle qui a disparu. La désinence -ora s'est répandue ensuite aussi aux noms masculins, en roumain, tout comme dans certains dialectes italiens.

Tous ces faits constituent les arguments en vertu desquels certains linguistes ont considéré que le neutre est le successeur direct du neutre latin (et par cela du neutre de l'indo-européen) ou qu'il représente une évolution interne du roumain mais ayant ses racines dans le latin (Meyer-Lübke $1895: 51-53$; Iordan $1956^{2}: 239$; Ivănescu 1957 : 299-313 ; Graur 1960 : 356 ; Ivănescu 1980 : 141-145, Fischer 1985 : 81-83 ; DGȘ $1997^{1}$ :324).

2. 4. 1. 4. Le neutre roumain - création entièrement roumaine. Puisque ni la forme, ni les détails concernant le sens du neutre roumain ne correspondent point au neutre latin, on a considéré que le neutre roumain est un fait purement et simplement roumain plutôt qu'un éventuel développement commun du roumain et des idiomes bulgares, macédoniens, ce genre n'étant en fait qu'un réarrangement originel du matériel linguistique roumain (Pătruț $1956: 29,30$; Rosetti 1978² : 601, 662-676, 668).

2. 4. 1. 5. Dynamique et avenir du neutre roumain. Ayant en vue un rapprochement plus serré du tronc commun des langues néolatines, où le neutre se manifeste de manière très périphérique et chétive, quelques linguistes ont nié la consistance du neutre et ont prédit son imminente disparition. Sans s'appuyer sur des données statistiques, on a soutenu carrément que : " le fondement matériel du neutre est faible et continue à s'affaiblir $»^{17}$ (Iordan $1956: 40$ ), que « Le neutre est en baisse aujourd'hui » ${ }^{18}$ (Graur $1973: 42$ ), que « le genre neutre commence à perdre du terrain par rapport au genre masculin $\gg^{19}$ (GLR 1966 : 57), ou bien que « dans le cadre de la concurrence entre le masculin et le neutre on constate une nette tendance de réduction du genre neutre $»^{20}(\text { Avram } 1997: 51)^{21}$.

Pour ceci on a invoqué les arguments suivants :

1) L'inconsistance sémantique de la classe - tous les neutres ne désignent pas seulement des inanimés (Iordan $1956: 40$ ) : animal « animal », dobitoc « bête », gasteropod « gastéropode », mamifer « mammifère », rumegător « ruminant », vertebrat « vertébré », série complétée par une suite de noms collectifs : batalion « bataillon », cârd « troupeau d'animaux », neam " peuple, nation », norod « peuple », pluton " peloton », popor « peuple », regiment « régiment », stol « volée, bande d'oiseaux », trib « tribu ».

17 « baza materială a neutrului este șubredă și se șubrezește mereu ».

18 « Astăzi neutrul este în general în scădere. ».

19 « genul neutru începe să piardă teren în comparație cu genul masculin ».

20 " în concurenta dintre masculin și neutru se constată o tendintă netă de restrângere a genului neutru».

21 Voir aussi Goga 1965 : 307, où on parle également de l'affaiblissement de la position du neutre. 
2) L'analogie avec les genres forts : puisque au singulier il vêtit la forme du masculin et au pluriel celle du féminin, le neutre a tendance à se reconstruire (Iordan 1956 : 40; Goga 1965 : 307 ; GLR 1966 : 57; Graur 1973 : 42; Iordan - Robu 1978 : 369; Avram $1997: 51)$ : a) une nouvelle forme masculine au pluriel, surtout dans la terminologie scientifique (sg. condensator « condensateur »-pl. condensatoare $\rightarrow$ condensatori, sg. corp « corps »-corpuri $\rightarrow$ corpi, sg. nucleu « noyau»-pl. nuclee $\rightarrow$ nuclei, sg. robinet « robinet »-pl. robinete $\rightarrow$ robineți), ou vice versa, b) une nouvelle forme féminine au singulier : $\mathrm{b}_{1}$ ) en - $\breve{a}$ (astm « asthme » $\rightarrow$ astmă , bonet « bonnet 》 $\rightarrow$ bonetă , basc « béret basque » $\rightarrow$ bască , beret « béret basque » $\rightarrow$ beretă $)$ ou $\left.\mathrm{b}_{2}\right)$ en $-e($ favor " faveur » $\rightarrow$ favoare, pustiu « désert » $\rightarrow$ pustie, rachiu « eau-de-vie » $\rightarrow$ rachie).

3) La concurrence qui surgit entre (Goga 1965 : 307) : a) quelques dérivés verbaux neutres et féminins formés à l'aide de suffixes différents : -ială (bâzâit / bâzâială « bourdonnement », croit / croială « coupe d'un habit », păruit / păruială « rixe »), -eală (bălăcit / bălăceală « pataugeage »), -tură (lătrat / lătrătură « aboiement »); b) les noms neutres issus des participes et les noms féminins issus des infinitifs (camuflaj-camuflare « camouflage »; finisaj - finisare « finissage »).

L'affaiblissement ou la dissolution du neutre en tant que classe destinée à rassembler les inanimés ne sont point soutenus par les constats statistiques (Diaconescu 1969 : 35). Par contre, on constate le dernier temps une tendance diamétralement opposée, à savoir une attraction croissante de la part du neutre pour les inanimés néologiques, surtout pour les anglicismes de l'informatique, ainsi que pour les sigles et les acronymes (Preda 2013 : 1075-1076) : computer-computere, desktop - desktopuri, discount-discounturi, dressing - dressinguri, drink-drinkuri, e-mail - e-mailuri, exit-poll - exit-polluri, fastfood-fast-fooduri, feedback-feedbackuri, flashback-flashbackuri, gadget-gadgeturi, grill - grilluri, hard - harduri, input - inputuri, poster - postere, prompter-promptere, server - servere, soft - softuri, starter - startere, taster-tastere ; $C D-C D$-uri, DVD DVD-uri, GPS - GPS-uri, SMS - SMS-uri, SUV-SUV-uri, WC-WC-uri...

Autrement dit, à la différence du français (et des autres langues romanes), le roumain dispose d'un nombre fort élevé de noms neutres, ce genre étant perçu comme très dynamique et très moderne : 1) un inventaire établi sur 4366 noms inanimés a recensé 1 470 noms neutres, 2674 noms féminins et seulement 222 noms masculins (DLRM 1958 apud Goga 1965 : 307) ; 2) une autre statistique a relevé 30, 1\% de noms neutres (395), $51,6 \%$ de noms féminins (678) et seulement 18, 2\% noms masculins (239) sur un total de 1312 noms (VRLR 1988 apud Găitănaru 2005 : 605).

3. Conclusions. Quoiqu'il en soit, étant donnée la complexité et le caractère peu logique de l'organisation du genre à l'intérieur de chacune des langues présentées ici, ainsi que la distribution asymétrique des noms en classes de genres entre le roumain et le français, seulement trois conclusions seront formulées à la fin de cet exposé.

1) Quoique langues apparentées génétiquement, le roumain et le français se distribuent de manière différente les noms en fonction du genre grammatical. Les lois internes sur lesquelles se sont forgées les deux langues, ainsi que l'influence du substrat ont été décisives dans cette répartition tellement divergente des noms en classes de genre.

Pour ce qui est des origines du neutre roumain, il est évident que c'est le latin qui a interféré avec le substrat thraco-dace et qu'il y avait quelques désinences très proches 
dans les deux langues (lat. -ora et le thraco-dace / alb. -(ë)na, -(ë)ra). Il paraît que ce modèle était bien incrusté dans le substrat, puisqu'il a survécu en albanais. Il paraît aussi que le substrat aurait dû consolider le modèle en -uri, qui est devenu très productif en roumain et qui s'est répandu ensuite parmi des mots entrés ultérieurement d'autres langues, étant également adopté par des mots féminins. Il serait plausible donc de considérer que le neutre roumain est une continuation du neutre latin tout en étant soutenu par le substrat thraco-dace (Găitănaru 2007 : 147).

Cependant ce type de neutre, interprété comme ambigenre ou genre hétérogène n'est pas totalement absent en français. Évidemment, sur ce mince corpus de noms « androgynes » attestés on est loin de pouvoir formuler la prétention d'admettre l'existence en français d'une catégorie / classe grammaticale à part entière et opposable à celles existant déjà.

2) Aussi curieux soit-il, le neutre roumain apparaît comme une classe homogène, vivante, dynamique et très moderne.

3) Une autre conclusion qui se dégage à la fin de ce survol de la problématique des genres dans les langues romanes et certaines langues balkaniques vise un aspect plus pratique car il renvoie directement à la didactique dès que ces langues entrent en contact.

L'acquisition du genre des noms roumains reste souvent embarrassante et malaisée à surmonter pour les locuteurs étrangers, parce que le roumain fonde l'opposition de genre sur trois termes : le masculin, le féminin et le neutre. La mauvaise ou la faible maitrise de la distribution des noms en fonction de la catégorie du genre reste une source incontournable d'erreurs interférentielles, dues à de fausses analogies, des interférences aussi bien inter- et intra-langue.

Il est évident que, comme il n'y a aucune logique en ce qui concerne l'attribution aux choses d'un genre quelconque et non pas d'un autre, cette répartition étant tout-àfait arbitraire et fortement liée aux caractéristiques de chaque langue, il faut apprendre directement les noms avec leur genre. C'est-à-dire les mémoriser purement et simplement d'une façon mécanique.

Certes, la démarche d'acquérir le genre n'est pas du tout la même chez locuteur natif et chez l'étranger (adulte) qui apprend n'importe quelle langue pourvue de genres. En règle générale, le natif intègre naturellement le genre grammatical de la plupart des noms grâce à une sorte de mécanisme interne, faisant inconsciemment appel à un complexe de critères (stimuli) inhérents à la structure des noms : a) contextuels (flexion / accord des déterminants-satellites : article, adjectif, pronom), b) phonético-graphiques et morphologiques (terminaisons), c) sémantiques (Jeanmaire, 2010). Au cas où toutes ces quelques règles ou lois empiriques paraissent insuffisantes, le natif est censé faire appel à des compétences linguistiques ou « encyclopédiques » qu'il n'arrive pas à maîtriser à l'oreille. Ce dernier subterfuge reste moins exploitable par le locuteur étranger.

\section{Bibliographie}

Arrivé, M. (1997). « Coup d'œil sur les conceptions du genre grammatical ». In Comptes rendus des séances de l'Académie des Inscriptions et Belles-Lettres, $141^{\mathrm{e}}$ année, $\mathrm{n}^{\circ}$ 1, pp. 81-96.

Avram, M. (19972). Gramatica pentru toti. Bucureşti : Editura Humanitas.

Bonfante, G. (2001). « Neutrul italienesc, românesc și albanez ». In Studii române. București : Editura Saeculum I. O., pp. 132-145. 
Brâncuş, G. (1963). « Genul neutru în albaneză ». In Studii și Cercetări Lingvistice, XIV, $\mathrm{n}^{\circ} 1$, pp. $75-85$. pp. 51-64.

Bujor, I. I. (1955). « Genul substantivelor în limba română ». In Limba română, IV, nº 6,

Coteanu, I. (1969). Morfologia numelui în protoromână. București : Editura Academiei. DAR (2003). Topciu, R. \& Melonashi, A. \& Topciu, L., Dictionar albanez român. Polirom. DGȘ (1997). Bidu-Vrânceanu, A. \& Călăraşu, C. \& Ionescu-Ruxăndoiu, L. \& Mancaș, M. \& Pană Dindelegan, G., Dicționar general de științe. Sțiințe ale limbii. București : Editura Științifică.

Diaconescu, P. (1969). « Sintagmatic și paradigmatic în structura genului din limba română ». In Studii si Cercetări Lingvistice, XX, n¹, pp. 23-40.

DLRM (1958). Dictionarul limbii române moderne. București : Editura academiei RPR.

Doca, Gh. \& Rochetti, A. (1992). Comprendre et pratiquer le roumain. Bucureşti - Paris : Editura Academiei Române - Université de la Sorbonne Nouvelle (Paris III).

Dubois, J. (1989). « Le genre dans les nom d'animaux ». In Genre et langage, Actes du colloque tenu à Paris X Nanterre les 14-16, LINX, C.R.L. - Université Paris X, pp. 87-92.

Elmiger, D. (2015). « Masculin, féminin : et le neutre ? Le statut du genre neutre en français contemporain et les propositions de 'neutralisation' de la langue ». In Implications philosophiques, 6 juin.

Feuillet, F. (2001). Aire linguistique balkanique (Chap. La caractéristique typologique de famille et d'aires linguistiques), Language Typology and Language Universals / Sprachtypologie und sprachliche Universalien / La typologie des langues et les universaux linguistiques, Tome 2 [Réd. : Martin Haspelmath]. Walter de Gruyter, pp. 1510-1528.

Fischer, I. (1985). Latina dunăreană. Introducere în istoria limbii române. București : Editura Științifică și Enciclopedică.

Găitănaru, M. (2005). « Genul neutru: structura etimologică şi constituirea claselor flexionare ». In Limba română - Aspecte sincronice și diacronice, Actele celui de al 5-lea Colocviu al Catedrei de Limba Română Universitatea din București, Facultatea de Litere. București : Editura Universității din București, pp. 603-607.

Găitănaru, M. (2007). « L'évolution du neutre roumain ». In Limba și literatura - repere identitare în context european, vol. I. Pitești : Editura Universității din Pitești, pp. 145-148.

GGSh (2002). Gramatika e gjuhës shkipe, [collectif], 1. Akademia e Shkencave e Shqipërisë, Instituti i Gjuhësisë dhe i Letërsisë, Akademia e shkencave e Shqipërisë.

GLR (1966). Gramatica limbii române, [colectiv], vol. I București : Editura Academiei RSR. Goga, E. (1965). « Situația genului neutru în limba română actuală». In Omagiu lui Alexandru Rosetti la 70 de ani. București: Editura Academiei RSR, pp. 307-310.

Goga, E. (1966). « Observații asupra genului comun în Romania ». In Studii și Cercetări Lingvistice, XVII, nr. 2, 1966, pp. 195-204.

Graur, Al. (1960). Studii de lingvistică generală. Bucureşti : Editura Academiei RSR. Graur, Al. (1973). Gramatica azi. București : Editura Științifică, București, 1968.

Grevisse, M. \& Goose A. Le Bon usage (électronique), De Boeck Supérieur [https://books. google.mk/books?id=lyPqRIMOxG8C\&redir_esc=y].

Guillard-Chamart, G. (2009). Un ou une? Règles internes du genre des noms en français. Éd. Édilivre Aparis.

Hořejši, Vl. (1957). « Problema substantivelor așa-zise 'neutre' în limba română în lumina legăturilor cu alte limbi ». In Studii și Cercetări Lingvistice, n 8, pp. 415-429.

Iordan, I. (1956²). Limba română contemporană. Editura Ministerului Învățământului.

Iordan, I. \& Robu, Vl. (1978). Limba română contemporană. București : Editura Didactică și Pedagogică.

Irimia, D. (1997). Gramatica limbii române. Iași : Editura Polirom.

Ivănescu, G. (1957). « Soarta neutrului latin clasic în latina populară și în limbile romanice ». In Studii și Cercetări Lingvistice, VIII, pp. 299-314. 
Ivănescu, G. (1980). Istoria limbii române. Iași : Editura Junimea.

Jeanmaire, G. (2010). « Vox populi vox Dei ? L'identification du genre grammatical en français ». In Langue française, $\mathrm{n}^{\circ} 168, \mathrm{pp}$. 71-86.

Kokona, V. (2002). Français-Albanais / Shqip-Frengjisht - Dictionnaire / Fjalor. [Tiranë] : Botimet Kokona.

LAROUSSE - Larousse.fr: encyclopédie et dictionnaires gratuits en ligne [http://www. larousse.fr/dictionnaires/francais].

Lécaillé, J.-L. (2010). Souvenirs d'enfance, EPI.

Livescu, Mi. (2009). Histoire interne du roumain : morphosyntaxe et syntaxe / Interne Sprachgeschichte des Rumänischen : Morphosyntax und Syntax, în Romanische Sprachgeschichte / Histoire linguistique de la Romania. 3. Teilband Tome 3. Walter de Gruyter, pp. 2646-2692. 173-179

Marchello-Nizia, Ch. (1989). « Le neutre et l'impersonnel ». In Linx, vol. 21, n 1, pp.

Meyer-Lübke, W. (1895). Grammaire des langues romanes [trad. fr. : Auguste Doutrepont, Georges Doutrepont]. Tome deuxième, Morphologie. Paris : Welter.

Michel, L. (2015). « Le 'neutre' d'une langue sans neutre ». In Implications philosophiques, 6 juin [http://www.implications-philosophiques.org/actualite/une/le-neutre-dune-langue-sans-neutre/].

Nandris, O. (1961). " Le Genre, ses réalisations et le genre personnel en roumain ». In Revue de linguistique romane, Tome XXV, pp. 47-74.

Papp, E. (1978). « Recunoașterea genului substantivelor românești după terminație ». In Limba română, XXVII, nr. 5, pp. 473-478.

Pătruț, I. (1956). « Despre genul 'neutru' în limba română ». In Cercetări de Lingvistică, L, n 1-4, pp. 29-40.

Pătruț, I. (1974). Studii de limba română și slavistică. Cluj : Editura Dacia.

Picoche, J. \& Marchello-Nizia Ch. (1989). Histoire de la langue française. Paris : Nathan.

Preda, Vl. (2013). «The present stage of lexical neologisms adaptation (with reference to Anglicisms and Americanisms) ». In The Proceedings of the International Conference Literature, Discourse and Multicultural Dialogue, Section Language and Discourse, [Éd. : Iulian Boldea], 1, Târgu-Mureș : Arhipelag XXI Press, pp. 1073-1077.

Rosetti, Al. (1978²). Istoria limbii române, de la origini până în secolul al XVII-lea. București : Editura pentru Literatură.

Sandfeld, K. (1930). Linguistique balkanique. Problèmes et résultats. Paris : Champion.

Sterpu, I. (2012). « Sur certaines difficultés de l'apprentissage du genre des noms roumains par les locuteurs natifs du français ». In Analele Universităţii « Ovidius » din Constanţa. Seria Filologie, $\mathrm{n}^{\circ}$ 23/1, pp. 91-104.

Tănase, A.-M. (1974). « Contributions à l'étude du 'genre neutre' en roumain ». In Actes du XIVe Congrès International de Linguistique et de Philologie Romanes, [Réd. : Alberto Vàrvaro], Napoli, pp. 331-338.

TLFi. Trésor de la Langue Française Informatisé [http://atilf.atilf.fr/tlf.htm]. 265-268.

Tøgeby, K. (1952). « Le problème du neutre roumain ». In Cahiers Sextil Pusçariu, 1, pp.

Veselaj, N. (2012). «Rreth identitetit të gjinisë asnjanëse në shqipen e sotme » (III). In Zemra Shqiptare 12.04 [http://www.zemrashqiptare.net/news/id_27136/Nuhi-Veselaj:-Rreth-identitetitt\%C3\%AB-gjinis\%C3\%AB-asnjan\%C3\%ABse-n\%C3\%AB-shqipen-e-sotme-\%28III\%29.html].

Violi, P. (1987). « Les origines du genre grammatical ». In Langages, 21e année, n 85, pp. 15-34.

VRLR (1988). Sala M. [coord. ], Bîrlădeanu, M. \& Iliescu, M. \& Macarie, L. \& Nichita, I. \& Ploae-Hanganu, M. \& Theban, M. \& Vintilă-Rădulescu, I., Vocabularul reprezentativ al limbilor romanice. București : Editura Științifică și Enciclopedică.

Габинский, М. А. (1956). « Автохтонные элементы в молдавском языке ». In Boūpocb языкознания, $\mathrm{n}^{\circ} 1$, pp. 85-93. 


\section{Constantin-Ioan MLADIN}

\section{NEUTRAL GENDER OF ROMANIAN NOUNS IN ROMANIAN AND BALKAN CONTEXT}

Summary: The aim of this paper is to reconsider one aspect of the Romanian morphology - the neutral gender of nouns.

Generally speaking, it has been accepted that of all the Neolatine languages it is only the Romanian language that has preserved neutral gender as a constant and productive grammatical category, and the neutrum has, as a subtype of person category, functioned as an agent re-motivating the natural gender. Moreover, and in spite of the pessimistic predictions of a few linguists of the last century who anticipated the disintegration of the neutral gender, the latter has proved to be a very dynamic and present category in modern Romanian. In order to better understand the state and origin of Romanian neutrum, in this paper we: a) retrace, in a concise and critical manner, the main theories of its possible origins (affiliation with Latin, relation with Albanian, influence of Old Church Slavonic), and b) compare situation in Romanian, a language simultaneously Romance and Balkan, and identical or similar situations of the other modern languages to which Romanian is historically related (Italian and Romansh, French, Spanish)

This research aims also at assessing the efficiency (real or alleged) of the different methods used for recognizing the Romanian neutrum, an aspect that is rather problematic and difficult to deal with when learning it as a foreign language (francophone learners being privileged in that respect).

Key words: grammatical gender (masculine, feminine, neutral), natural gender, Albanian, Spanish, French, Italian, Latin, Romanian, Romansh, Old Church Slavonic. 